\title{
Parametric amplification of matter waves in dipolar spinor Bose-Einstein condensates
}

\author{
F. Deuretzbacher, ${ }^{1,}{ }^{*}$ G. Gebreyesus, ${ }^{1}$ O. Topic, ${ }^{2}$ M. Scherer, ${ }^{2}$ B. Lücke, ${ }^{2}$ W. Ertmer, ${ }^{2}$ J. Arlt, ${ }^{3}$ C. Klempt ${ }^{2}$ and L. Santos ${ }^{1}$ \\ ${ }^{1}$ Institut für Theoretische Physik, Leibniz Universität Hannover, Appelstr. 2, D-30167, Hannover, Germany \\ ${ }^{2}$ Institut für Quantenoptik, Leibniz, Universität Hannover, Welfengarten 1, D-30167 Hannover, Germany \\ ${ }^{3}$ QUANTOP, Danish National Research Foundation Center for Quantum Optics, Department of Physics and Astronomy, \\ Aarhus University, Ny Munkegade 120, DK-8000 Aarhus C, Denmark
}

(Received 12 May 2010; published 10 November 2010)

\begin{abstract}
Spin-changing collisions may lead under proper conditions to the parametric amplification of matter waves in spinor Bose-Einstein condensates. Magnetic dipole-dipole interactions, although typically very weak in alkalimetal atoms, are shown to play a very relevant role in the amplification process. We show that these interactions may lead to a strong dependence of the amplification dynamics on the angle between the trap axis and the magnetic-field orientation. We analyze as well the important role played by magnetic-field gradients, which also modify strongly the amplification process. Magnetic-field gradients, hence, must be carefully controlled in future experiments, in order to observe clearly the effects of the dipolar interactions in the amplification dynamics.
\end{abstract}

DOI: 10.1103/PhysRevA.82.053608

PACS number(s): 67.85.Fg, 67.85.De, 67.85.Hj, 75.50.Mm

\section{INTRODUCTION}

Spinor Bose-Einstein condensates (BECs), formed by atoms with various available Zeeman states, have attracted a large attention in recent years, mostly motivated by the rich physics resulting from the interplay between internal and external degrees of freedom. In addition to a wealth of possible ground-state phases [1-4], the spinor dynamics has been at the focus of major interest [5-7]. This dynamics results from spin-changing collisions, which coherently re-distribute the populations among the different Zeeman states. Interestingly, spin-changing collisions are typically characterized by a very low energy scale much lower than the chemical potential in the condensate. As a result of that, the spinor dynamics in alkali-metal gases may be extraordinarily sensitive to other small energy scales.

Up until very recently, only short-range interactions have played a role in typical experiments in ultracold gases. Recent experiments have started to unveil the rich physics resulting from the dipole-dipole interactions (DDI) [8,9]. This is particularly the case of chromium, which presents a relatively large magnetic dipole moment, $\mu=6 \mu_{B}$. Remarkable effects of the magnetic DDI have been reported in recent experiments on chromium BEC [10-13]. Alkali-metal atoms, on the contrary, present a much lower magnetic dipole moment, $\mu=\mu_{B} / 2$, and hence they are not usually expected to show any trace of the DDI unless short-range interactions are switched off by means of Feshbach resonances [14,15]. However, as mentioned earlier, the spin-changing collisions in alkali-metal spinor BECs (in particular $F=1{ }^{87} \mathrm{Rb}$ ) are remarkably low energetic. As a result, spinor dynamics is very sensitive to magnetic DDI, in spite of the very low magnetic dipole moment. Recent experiments [16] have shown that the DDI may induce magnetization patterns in $F=1{ }^{87} \mathrm{Rb}$ BECs.

Spinor dynamics is particularly interesting for the case of condensates initially prepared in the $m=0$ Zeeman sublevel. In that case, spin-changing collisions may lead to correlated Einstein-Podolsky-Rosen (EPR) pairs in $m= \pm 1[17,18]$, in a

\footnotetext{
*fdeuretz@itp.uni-hannover.de
}

process which closely resembles parametric down-conversion in nonlinear optics [19]. As a result, spinor condensates may act as parametric amplifiers of matter waves [20-22], opening interesting perspectives for the creation of nonclassical states of matter based on spinor BECs. Recently we have shown that the interplay between trapping potential, quadratic Zeeman effect (QZE), and spin-changing collisions crucially determines the amplification gain [21] and its sensitivity with respect to quantum spin fluctuations [22].

In this paper we show that the amplification dynamics may be extremely sensitive to the DDI. As a result of that, the amplification of EPR-like pairs is strongly modified by the relative orientation between the applied magnetic field and the trap axis. We analyze in detail this dependence, as well as the effects of magnetic-field gradients. We show that these gradients must be carefully controlled, since uncontrolled gradients may obscure the expected DDI effects. We finally comment on experimental requirements.

The structure of the paper is as follows. In Sec. II we present the system considered and the corresponding Hamiltonian. The linear regime is discussed in Sec. III. An intuitive qualitative picture of the effects of the DDI in the amplification dynamics is discussed in Sec. IV. In Sec. V we introduce the main formalism to analyze the amplification dynamics in the presence of DDI, whereas the corresponding numerical results are presented in Sec. VI. The effects of the magneticfield gradient are analyzed in Sec. VII. Finally we discuss experimental requirements and summarize our conclusions in Sec. VIII.

\section{HAMILTONIAN}

In the following we consider a spin-1 Bose gas (e.g., $F=1$ ${ }^{87} \mathrm{Rb}$ ), with Zeeman components $m=0, \pm 1$, confined in a dipole trap in the presence of an external magnetic field (which we assume as oriented along the $z$ axis). The system is described by the Hamiltonian $\hat{H}=\hat{H}_{0}+\hat{H}_{\text {sr }}+\hat{H}_{\text {dd }}$. In this Hamiltonian, the single-particle physics is described by

$\hat{H}_{0}=\int d^{3} r \sum_{m} \hat{\psi}_{m}^{\dagger}(\vec{r})\left[-\frac{\hbar^{2} \Delta}{2 M}+V(\vec{r})+E_{Z}(m)\right] \hat{\psi}_{m}(\vec{r})$, 
where $\hat{\psi}_{m}$ annihilates bosons with spin projection $m$. The trapping potential is of the form $V(\vec{r})=\frac{M}{2}\left[\omega_{\perp}^{2}\left(x^{2}+y^{\prime 2}\right)+\right.$ $\left.\omega_{\|}^{2} z^{\prime 2}\right]$, where $M$ is the atomic mass, $\omega_{\|} \ll \omega_{\perp}$ are the trap frequencies (cigar-shaped trap), and $y^{\prime}=(\cos \vartheta) y+\sin \vartheta z$, $z^{\prime}=-(\sin \vartheta) y+\cos \vartheta z$, with $\vartheta$ the angle between the weak trap axis ( $z^{\prime}$ axis) and the magnetic-field orientation. This angle will play a crucial role in our discussion of the effects of the DDI.

The Zeeman energy for the $m$ component is of the form,

$$
E_{Z}(m) \simeq(p+\vec{\nabla} p \cdot \vec{r}) m+q m^{2},
$$

where $p=g_{L} \mu_{B} B_{0}$ characterizes the linear Zeeman effect (LZE) for a homogeneous magnetic field $B_{0}$, with $g_{L}$ the Landé factor $\left(g_{L}=-1 / 2\right.$ for $\left.F=1{ }^{87} \mathrm{Rb}\right)$ and $\mu_{B}$ the Bohr magneton. The quadratic Zeeman effect (QZE) is characterized by a constant $q$, which in principle depends as $q=\mu_{B}^{2} B_{0}^{2} /\left(8 C_{\mathrm{hfs}}\right)$ on the hyperfine coupling strength $C_{\mathrm{hfs}}(\approx h \times 3.4 \mathrm{GHz}$ for ${ }^{87} \mathrm{Rb}$ ), but may also be externally modified using optical or microwave dressing [23,24]. Additionally, we allow for a magnetic-field gradient $B=B_{0}+\vec{\nabla} B \cdot \vec{r}$, leading to an energy shift $m \vec{\nabla} p \cdot \vec{r}$, which plays a relevant role below.

The short-range interactions are given by

$$
\hat{H}_{\mathrm{sr}}=\frac{1}{2} \int d^{3} r \sum_{\substack{m, m^{\prime} \\ \bar{m}^{\prime}, \bar{m}}} \hat{\psi}_{m}^{\dagger}(\vec{r}) \hat{\psi}_{m^{\prime}}^{\dagger}(\vec{r}) U_{m, m^{\prime}}^{\bar{m}^{\prime}, \bar{m}} \hat{\psi}_{\bar{m}^{\prime}}(\vec{r}) \hat{\psi}_{\bar{m}}(\vec{r}),
$$

with $U_{m, m^{\prime}}^{\bar{m}^{\prime}, \bar{m}} \equiv U_{0} \delta_{m, \bar{m}} \delta_{m^{\prime}, \bar{m}^{\prime}}+U_{1} \vec{f}_{m \bar{m}} \cdot \vec{f}_{m^{\prime} \bar{m}^{\prime}}$, where $\vec{f}_{m m^{\prime}}=$ $\left(f_{m m^{\prime}}^{x}, f_{m m^{\prime}}^{y}, f_{m m^{\prime}}^{z}\right)^{T}$, with $f_{x, y, z}$ the spin-1 matrices. $U_{0}=$ $\left(g_{0}+2 g_{2}\right) / 3$ and $U_{1}=\left(g_{2}-g_{0}\right) / 3$ are, respectively, the coupling constants for spin-independent and spin-dependent interactions, where $g_{F}=4 \pi \hbar^{2} a_{F} / M$, with $a_{F}$ the $s$-wave scattering length for the channel with total spin $F$. Note that the short-range interactions preserve the total spin projection, but this may be done in two crucially different ways, either by preserving the individual spin projections (spin-preserving collisions) or by modifying the individual projections while preserving the total one (spin-changing collisions).

Finally, the magnetic dipole-dipole interaction is given by

$$
\begin{aligned}
\hat{H}_{\mathrm{dd}}= & \frac{1}{2} \int d^{3} r \int d^{3} r^{\prime} \sum_{\substack{m, m^{\prime} \\
\bar{m}^{\prime}, \bar{m}}} \hat{\psi}_{m}^{\dagger}(\vec{r}) \hat{\psi}_{m^{\prime}}^{\dagger}\left(\vec{r}^{\prime}\right), \\
& W_{m, m^{\prime}}^{\bar{m}^{\prime}, \bar{m}}\left(\vec{r}-\vec{r}^{\prime}\right) \hat{\psi}_{\bar{m}^{\prime}}\left(\vec{r}^{\prime}\right) \hat{\psi}_{\bar{m}}(\vec{r}),
\end{aligned}
$$

with

$$
\begin{aligned}
& W_{m, m^{\prime}}^{\bar{m}^{\prime}, \bar{m}}\left(\vec{r}-\vec{r}^{\prime}\right) \\
& \quad \equiv \frac{d^{2}}{\left|\vec{r}-\vec{r}^{\prime}\right|^{3}}\left[\vec{f}_{m \bar{m}} \cdot \vec{f}_{m^{\prime} \bar{m}^{\prime}},-3\left(\vec{f}_{m \bar{m}} \cdot \vec{u}_{r}\right)\left(\vec{f}_{m^{\prime} \bar{m}^{\prime}} \cdot \vec{u}_{r}\right)\right],
\end{aligned}
$$

where $d^{2}=\mu_{0} g_{L}^{2} \mu_{B}^{2} /(4 \pi), \mu_{0}$ is the vacuum permeability, and $\vec{u}_{r}=\left(\vec{r}-\vec{r}^{\prime}\right) /\left|\vec{r}-\vec{r}^{\prime}\right|$. Contrary to the short-range interactions the DDI may violate, in principle, the conservation of the total spin projection (they may induce the equivalent of the Einstein-de Haas effect [25,26]). However, the associated change in LZE is typically, even for very low magnetic fields, orders of magnitude larger than any energy in the system and hence these spin-violating processes can be safely considered as suppressed.
Since short-range interactions preserve spin projection, and so do in practice DDI as well, the homogeneous LZE $(\mathrm{pm})$ is preserved and it may be gauged out, playing no role in the dynamics discussed in the following. The same argument cannot be, however, employed with the magnetic-field gradient which may play a significant role in the spinor dynamics [27], as discussed in Sec. VII.

\section{LINEAR REGIME}

In the following we are interested in the first stages (linear regime) of the spinor dynamics of a spin-1 BEC initially prepared in the $m=0$ sublevel, after quenching $q$ into the unstable regime. This dynamics, induced by spin-changing collisions, is characterized by the correlated creation of atomic pairs in $m= \pm 1$. In this section, and for the sake of simplicity, we do not consider magnetic-field gradients, which will be introduced in Sec. VII.

Before quenching $q$, the BEC is prepared in the $m=0$ component. The initial scalar wave function $\psi_{0}$ of the BEC in $m=0$ and the corresponding chemical potential $\mu$ may be obtained from the time-independent Gross-Pitaevskii equation:

$$
\left[-\frac{\hbar^{2}}{2 M} \Delta+V(\vec{r})+U_{0} n_{0}(\vec{r})\right] \psi_{0}(\vec{r})=\mu \psi_{0}(\vec{r}),
$$

with $n_{0}(\vec{r})=\left|\psi_{0}(\vec{r})\right|^{2}$, and $\int d^{3} r\left|\psi_{0}(\vec{r})\right|^{2}=N$.

The first stages of the spinor dynamics may be described by means of a Bogoliubov approximation:

$$
\left(\hat{\psi}_{1}, \hat{\psi}_{0}, \hat{\psi}_{-1}\right)^{T}=\left[\left(0, \psi_{0}, 0\right)^{T}+\left(\delta \hat{\psi}_{1}, \delta \hat{\psi}_{0}, \delta \hat{\psi}_{-1}\right)^{T}\right] e^{-i \mu t},
$$

where we consider small fluctuations of the spinor field operator $\left\{\delta \hat{\psi}_{m}\right\}$, such that $\left|\psi_{0}\right|^{2} \gg \sum_{m}\left\langle\delta \hat{\psi}_{m}^{\dagger} \delta \hat{\psi}_{m}\right\rangle$.

\section{A. Hamiltonian without dipole-dipole interactions}

We consider first the Hamiltonian without DDI. Inserting (7) into the grand canonical potential $\hat{H}_{0}+\hat{H}_{\mathrm{sr}}-\mu \hat{N}$ [with $\hat{N}=\int d^{3} r \sum_{m} \hat{\psi}_{m}^{\dagger}(\vec{r}) \hat{\psi}_{m}(\vec{r})$ the total particle number], and keeping terms up to second order in $\delta \hat{\psi}_{m}$, we obtain an effective Hamiltonian for $\delta \hat{\psi}_{ \pm 1}$ of the form,

$$
\begin{aligned}
\hat{H}_{1}= & \sum_{m= \pm 1} \int d^{3} r \delta \hat{\psi}_{m}^{\dagger}\left(\hat{H}_{\mathrm{eff}}+q\right) \delta \hat{\psi}_{m} \\
& +U_{1} \int d^{3} r n_{0}\left(\delta \hat{\psi}_{1}^{\dagger} \delta \hat{\psi}_{-1}^{\dagger}+\delta \hat{\psi}_{1} \delta \hat{\psi}_{-1}\right)
\end{aligned}
$$

Note that in the linear regime the fluctuations $\delta \hat{\psi}_{ \pm 1}$ are decoupled from the density and phase fluctuations $\delta \hat{\psi}_{0}$ of the $m=0$ BEC (which may be excited during the preparation process). In the previous expression we have introduced $\hat{H}_{\text {eff }} \equiv-\hbar^{2} \Delta / 2 M+V_{\text {eff }}(\vec{r})$, where

$$
V_{\mathrm{eff}}(\vec{r})=V(\vec{r})+\left(U_{0}+U_{1}\right) n_{0}(\vec{r})-\mu
$$

may be understood as the effective trapping potential felt by the \pm 1 fluctuations on top of the $m=0$ BEC. It contains the mean-field potential $\left(U_{0}+U_{1}\right) n_{0}$, which originates from spin-preserving collisions of \pm 1 atoms with the BEC in $m=0$. 
Note that in the Thomas-Fermi regime $\mu=V(\vec{r})+U_{0} n_{0}(\vec{r})$. In that regime, $V_{\text {eff }}(\vec{r})=U_{1} n_{0}(\vec{r})$ within the BEC region, and $V_{\text {eff }}=V(\vec{r})-\mu$ outside.

The second line in Eq. (8) originates from spin-changing collisions, which convert $m=0$ atoms into \pm 1 -atom pairs and vice versa. Interestingly, this process resembles parametric down-conversion in optical parametric amplifiers [19]. Indeed, if the $m=0 \mathrm{BEC}$ is unstable after the quench of $q$, spinchanging collisions lead to an exponential amplification of the population in $m= \pm 1$ [20-22].

\section{B. Dipole-dipole interactions}

As mentioned earlier, spin-changing collisions are typically characterized by a very low-energy scale. As a result, the spinor physics is highly sensitive to other very low energy scales, including the rather weak magnetic DDI in alkali-metal gases. This sensitivity has been recently demonstrated in experiments on the formation of spatial magnetization patterns in spinor Rb BECs [16]. As we show in the following sections, the exponential amplification of the population in $m= \pm 1$ following a quench in $q$ may also be very significantly modified by the DDI.

Although there is no DDI contribution in the GP Eq. (6), since $\vec{f}_{00}=\overrightarrow{0}$, there is, however, an important contribution to the effective Hamiltonian for $\delta \hat{\psi}_{ \pm 1}$ which we obtain after inserting (7) in $\hat{H}_{\mathrm{dd}}$ and linearizing:

$$
\begin{aligned}
\hat{H}_{1, \mathrm{dd}}= & \int d^{3} r \int d^{3} r^{\prime} \psi_{0}(\vec{r}) \psi_{0}\left(\vec{r}^{\prime}\right) V_{\mathrm{dd}}\left(\vec{r}-\vec{r}^{\prime}\right) \\
& \times\left[\delta \hat{\psi}_{1}^{\dagger}(\vec{r}) \delta \hat{\psi}_{1}\left(\vec{r}^{\prime}\right)+\delta \hat{\psi}_{-1}^{\dagger}(\vec{r}) \delta \hat{\psi}_{-1}\left(\vec{r}^{\prime}\right)\right. \\
& \left.+\delta \hat{\psi}_{1}^{\dagger}(\vec{r}) \delta \hat{\psi}_{-1}^{\dagger}\left(\vec{r}^{\prime}\right)+\delta \hat{\psi}_{1}(\vec{r}) \delta \hat{\psi}_{-1}\left(\vec{r}^{\prime}\right)\right],
\end{aligned}
$$

with $V_{\mathrm{dd}}(\vec{r})=\frac{d^{2}}{2|\vec{r}|^{5}}\left(3 z^{2}-|\vec{r}|^{2}\right)$. In Eq. (10) we have neglected terms related to scattering processes which do not preserve the total spin projection since, as mentioned in Sec. II, the associated change in LZE suppresses spin-violating processes even for very low magnetic fields. Note that the third line of $\hat{H}_{1, \mathrm{dd}}$ contains as well a parametric amplification term.

\section{QUALITATIVE PICTURE OF THE EFFECT OF THE DIPOLE-DIPOLE INTERACTIONS ON THE AMPLIFICATION DYNAMICS}

The effects of the DDI on the amplification dynamics may be qualitatively understood from a simplified model. In homogeneous space ( $V=0$, constant $n_{0}, \mu=U_{0} n_{0}$ ) we may introduce the Fourier transform $\hat{\eta}_{m}(\vec{k})=\int d^{3} r \delta \hat{\psi}_{m}(\vec{r}) e^{-i \vec{k} \cdot \vec{r}}$, which allows one to write $\hat{H}_{1}$ in the simplified form [28] $H_{1}^{\text {hom }}=\int \hat{h}_{k} d^{3} k /(2 \pi)^{3}$, where

$$
\begin{aligned}
\hat{h}_{k}= & \sum_{m= \pm 1}\left(E_{k}+q-q_{c r}\right) \eta_{m}^{\dagger}(\vec{k}) \eta_{m}(\vec{k}) \\
& -q_{c r}\left[\hat{\eta}_{1}^{\dagger}(\vec{k}) \hat{\eta}_{-1}^{\dagger}(-\vec{k})+\hat{\eta}_{1}(\vec{k}) \hat{\eta}_{-1}(-\vec{k})\right],
\end{aligned}
$$

with $E_{k}=\hbar^{2} k^{2} / 2 M$ and $q_{c r}=-U_{1} n_{0}$. Note that for $F=1$ ${ }^{87} \mathrm{Rb} U_{1}<0$, and hence $q_{c r}>0$ (we consider in the following this case, although for the $F=2$ case the sign is the opposite).
This Hamiltonian, which may be easily diagonalized for each momentum $\vec{k}$, possesses eigenenergies of the form,

$$
\lambda^{ \pm}(\vec{k})=\sqrt{\left(E_{k}+q-q_{c r}\right)^{2}-q_{c r}^{2}} .
$$

Note that if $\operatorname{Im}\left[\lambda^{ \pm}(\vec{k})\right]>0$ for some eigenenergy, then there is an exponential growth of spin excitations, which leads to a correlated pair creation in $m= \pm 1$. This instability is best characterized by the instability rate $\Lambda=\max \left\{\operatorname{Im}\left[\lambda_{ \pm}(\vec{k})\right]\right\}$. It is straightforward to show that the $m=0$ BEC becomes unstable for $q<2 q_{c r}$. The instability rate rises between $q_{c r}<q<$ $2 q_{c r}$ acquiring its maximal value $\Lambda=q_{c r}$ at $q=q_{c r}$. For $q<q_{c r}, \Lambda=q_{c r}$ in the homogeneous case (in the inhomogeneously trapped case the instability rate $\Lambda$ presents significant modulations which are responsible for the multiresonant $q$ dependence of the amplification dynamics recently observed experimentally [21]).

In the following we apply a similar formalism to the DDI term $\hat{H}_{1, \mathrm{dd}}$. We introduce the Fourier transformation of $V_{\mathrm{dd}}(\vec{r})$ to obtain $\tilde{V}_{\mathrm{dd}}(\vec{k})=U_{\mathrm{dd}}\left(1-3 \cos ^{2} \theta\right)$, where $U_{\mathrm{dd}}=2 \pi d^{2} / 3$ and $\theta$ is the angle between $\vec{k}$ and the dipole orientation ( $z$ axis). Using convolution theorem, and since we assume $n_{0}$ as constant, we may then rewrite $\hat{H}_{1, \mathrm{dd}}$ in the form,

$$
\begin{aligned}
\hat{H}_{1, \mathrm{dd}}^{\mathrm{hom}}= & \int \frac{d^{3} k}{(2 \pi)^{3}} n_{0} \tilde{V}_{\mathrm{dd}}(\vec{k})\left\{\sum_{m= \pm 1} \hat{\eta}_{m}^{\dagger}(\vec{k}) \hat{\eta}_{m}(\vec{k})\right. \\
& \left.+\hat{\eta}_{1}^{\dagger}(\vec{k}) \hat{\eta}_{-1}(-\vec{k})^{\dagger}+\hat{\eta}_{1}(\vec{k}) \hat{\eta}_{-1}(-\vec{k})\right\} .
\end{aligned}
$$

Note that adding $H_{1, \text { dd }}$ to $H_{1}$ results in a similar form as that of $\hat{H}_{1}$ but with an effective $q_{c r}^{\text {eff }}(\theta)=q_{c r}-n_{0} \tilde{V}_{\mathrm{dd}}(\vec{k})$. Note that, due to the anisotropy of the DDI, $q_{c r}^{\text {eff }}$ depends on the angle $\theta$. The effects of the trap geometry may be qualitatively understood from this $\theta$ dependence. For an axisymmetric trap the dominant momenta are those along the tightest direction. If the dipole orientation is perpendicular to the trap axis, then the dominant $\vec{k}$ will then be those with $\theta=0$, and hence $q_{c r}^{\text {eff }} \simeq q_{c r}+2 n_{0} U_{\mathrm{dd}}$. On the contrary, if the dipole orientation is parallel to the trap axis, the dominant momenta will be those with $\theta=\pi / 2$, and $q_{c r}^{\text {eff }} \simeq q_{c r}-n_{0} U_{\mathrm{dd}}$. Since $\Lambda \simeq q_{c r}^{\text {eff }}$, we hence expect an enhancement of the instability for a magnetic-field orientation perpendicular to the trap axis, and a reduced instability for a parallel orientation. Although the DDI in alkali-metal atoms is typically very weak, the spin-changing collisions are very weak as well. In particular, in $F=1{ }^{87} \mathrm{Rb}$ the strength of the DDI is quite significant compared to the strength of the spin-changing collisions, $\left|U_{\mathrm{dd}} / U_{1}\right| \approx 0.2$. As a result, the DDI modification of the instability rate is expected to lead to a marked orientation dependence of the amplification dynamics. In the following sections we show that this is indeed the case when considering realistic trapped cases.

\section{AMPLIFICATION DYNAMICS IN TRAPPED DIPOLAR CONDENSATES}

Although the homogeneous model discussed before allows for a simplified intuitive understanding of the major effects of the DDI in the amplification process, a quantitative analysis of realistic experimental situations may be just achieved by 
properly considering the inhomogeneous trapping, and the corresponding inhomogeneous density $n_{0}(\vec{r})$ of the $m=0$ BEC. In this section we introduce the basic formalism which we follow for the analysis of the amplification dynamics characterizing the spinor physics in the linear regime.

The analysis of the spinor dynamics is significantly simplified by considering the eigenfunctions and eigenenergies of $\hat{H}_{\text {eff }}, \hat{H}_{\text {eff }} \phi_{n}(\vec{r})=\epsilon_{n} \phi_{n}(\vec{r})$, and expanding the field operators in the basis of these eigenstates $\delta \hat{\psi}_{m}(\vec{r})=\sum_{n} \phi_{n}(\vec{r}) \hat{a}_{n m}$. We may then rewrite

$$
\begin{aligned}
\hat{H}_{1}+H_{1, \mathrm{dd}}= & \sum_{n n^{\prime}}\left[\left(\epsilon_{n}+q\right) \delta_{n n^{\prime}}+B_{n n^{\prime}}\right] \sum_{m= \pm 1} \hat{a}_{n m}^{\dagger} \hat{a}_{n^{\prime} m} \\
& +\sum_{n n^{\prime}}\left(A_{n n^{\prime}}+B_{n n^{\prime}}\right)\left(\hat{a}_{n 1}^{\dagger} \hat{a}_{n^{\prime}-1}^{\dagger}+\hat{a}_{n 1} \hat{a}_{n^{\prime}-1}\right),
\end{aligned}
$$

where $A_{n n^{\prime}}=U_{1} \int d^{3} r n_{0} \phi_{n} \phi_{n^{\prime}}$ characterizes the effects of the short-range spin-changing collisions, whereas the effects of the DDI are given by

$$
B_{n n^{\prime}}=\int d^{3} r \int d^{3} r^{\prime} F_{n}(\vec{r}) V_{\mathrm{dd}}\left(\vec{r}-\vec{r}^{\prime}\right) F_{n^{\prime}}\left(\vec{r}^{\prime}\right),
$$

where $F_{n}(\vec{r})=\psi_{0}(\vec{r}) \phi_{n}(\vec{r})$. The matrix elements $B_{n n^{\prime}}$ are most efficiently calculated in $\vec{k}$ space according to

$$
B_{n n^{\prime}}=\int \frac{d^{3} k}{(2 \pi)^{3}} \tilde{F}_{n}(\vec{k}) \tilde{V}_{\mathrm{dd}}(\vec{k}) \tilde{F}_{n^{\prime}}(\vec{k}),
$$

where $\tilde{F}(\vec{k})$ is the Fourier transform of $F(\vec{r})$.

Equation (14) is solved by the multimode Bogoliubov ansatz,

$$
\hat{\alpha}_{v}^{ \pm}=\sum_{n}\left(u_{v n}^{ \pm} \hat{a}_{n 1}+v_{v n}^{ \pm} \hat{a}_{n-1}^{\dagger}\right),
$$

where $\hat{\alpha}_{v}^{ \pm}$satisfy $\left[\hat{\alpha}_{v}^{ \pm}, \hat{H}_{1}+H_{1, \mathrm{dd}}\right]=\lambda_{v}^{ \pm} \hat{\alpha}_{v}^{ \pm}$, which leads to the eigenvalue equation:

$$
\mathbf{C}\left(\begin{array}{c}
\vec{u}_{v}^{ \pm} \\
\vec{u}_{v}^{ \pm}
\end{array}\right)=\lambda_{v}^{ \pm}\left(\begin{array}{c}
\vec{u}_{v}^{ \pm} \\
\vec{u}_{v}^{ \pm}
\end{array}\right)
$$

where $\vec{u}_{v}^{ \pm T} \equiv\left\{u_{v 1}^{ \pm}, u_{v 2}^{ \pm}, \ldots\right\}$ (and similarly for $\vec{v}_{v}^{ \pm}$) and

$$
\mathbf{C}=\left[\begin{array}{cc}
\mathbf{E}+q \mathbf{1}+\mathbf{B} & -\mathbf{A}+\mathbf{B} \\
\mathbf{A}+\mathbf{B} & -\mathbf{E}-q \mathbf{1}-\mathbf{B}
\end{array}\right],
$$

with $E_{n n^{\prime}}=\epsilon_{n} \delta_{n n^{\prime}}, \mathbf{1}$ the identity matrix, and $\mathbf{A}$ (B) the matrix with components $A_{n n^{\prime}}\left(B_{n n^{\prime}}\right)$. From the Heisenberg equations of motion $\hat{\alpha}_{v}^{ \pm}(t)=\hat{\alpha}_{v}^{ \pm}(0) e^{-i \lambda_{v}^{ \pm} t / \hbar}$. Note that, as for the homogeneous case, if $\operatorname{Im}\left(\lambda_{v}^{ \pm}\right)>0$ for some eigenenergy, then there is an exponential growth of correlated pairs in $m= \pm 1$. As for the homogeneous case, this instability is best characterized by the instability rate $\Lambda=\max \left\{\operatorname{Im}\left(\lambda_{v}^{ \pm}\right)\right\}$.

The time evolution of $\hat{a}_{n, \pm 1}$ is then easily obtained:

$$
\left[\begin{array}{c}
\left\{\hat{a}_{n 1}(t)\right\} \\
\left\{\hat{a}_{n-1}^{\dagger}(t)\right\}
\end{array}\right]=\mathbf{U}(t)\left[\begin{array}{c}
\left\{\hat{a}_{n 1}(0)\right\} \\
\left\{\hat{a}_{n-1}^{\dagger}(0)\right\}
\end{array}\right],
$$

with $\mathbf{U}=\mathbf{M}^{-1} e^{-i \boldsymbol{\Lambda} t / \hbar} \mathbf{M}$, where $\mathbf{M}$ is the matrix of eigenvectors obtained after solving Eq. (18) and $\boldsymbol{\Lambda}$ the corresponding diagonal matrix of eigenvalues.
As mentioned earlier, the atoms are initially prepared in the $m=0$ sublevel. However, a slightly imperfect preparation may lead to a nonzero population of $N_{s} m= \pm 1$ pairs in the original BEC [22]. These spurious atoms (which from now on are called classical seed) share the same wave function $\psi_{0}(\vec{r})$ as the $m=0$ atoms. Denoting $\chi_{n}=\int d^{3} r \psi_{0}(\vec{r}) \phi_{n}(\vec{r}) / \sqrt{N}$, we may then easily express the population $P_{m}=\sum_{n}\left\langle\hat{a}_{n, m}^{\dagger} \hat{a}_{n, m}\right\rangle$ in the form $P_{m}(t)=P_{C}(t)+P_{Q}(t)$, where

$$
P_{C}(t)=N_{s} \vec{\chi} \cdot\left(\mathbf{O}^{\dagger} \mathbf{O}+\widetilde{\mathbf{O}}^{\dagger} \widetilde{\mathbf{O}}\right) \vec{\chi}
$$

denotes the population triggered by the classical seed, and

$$
P_{Q}(t)=\operatorname{Tr}\left(\widetilde{\mathbf{O}}^{\dagger} \widetilde{\mathbf{O}}\right)
$$

denotes the population induced by quantum fluctuations (i.e., when $N_{s}=0$ ). In the previous expressions, the matrices $\mathbf{O}$ and $\widetilde{\mathbf{O}}$ are the upper left and upper right part of the time evolution matrix $\mathbf{U}(t)$ and $\vec{\chi}=\left(\chi_{1}, \chi_{2}, \ldots\right)^{T}$.

\section{DIPOLE-INDUCED ORIENTATION DEPENDENCE OF THE AMPLIFICATION DYNAMICS}

In this section we employ the formalism discussed in Sec. V to study the effects of the DDI in the amplification dynamics. We shall show that due to the DDI the amplification may be markedly dependent on the relative orientation between the trap axis and the external magnetic field.

In our numerical calculations we have considered realistic experimental conditions, with $N=10^{5} F=1{ }^{87} \mathrm{Rb}$ atoms in a cigar-shaped harmonic potential with $\omega_{\perp}=2 \pi \times 200 \mathrm{~Hz}$ and $\omega_{\|}=2 \pi \times 40 \mathrm{~Hz}$. As mentioned earlier, we consider the atoms as initially prepared in $m=0$ with possibly an initial spurious classical seed (which we typically consider as $N_{s}=2$, a typical value expected from previous experimental results [22]). At $t=0$ the QZE energy $q$ is set to a given value within the instability regime. We monitor the subsequent evolution of the populations $P_{ \pm 1}(t)$ obtained from Eqs. (21) and (22) as a function of $q$ and the relative angle $\theta$ between the trap axis and the external magnetic field.

Figure 1 shows the dependence of the instability rate $\Lambda$ as a function of $q$ for $\theta=0, \theta=\pi / 2$, and without DDI. Note that for all cases, the instability rate experiences a maximum

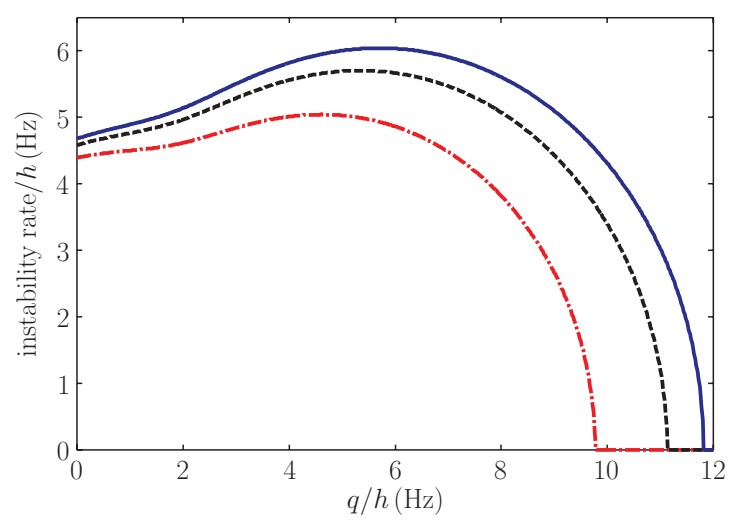

FIG. 1. (Color online) Instability rate $\Lambda$ as a function of $q$ for $N=10^{5}, \omega_{\perp}=2 \pi \times 200 \mathrm{~Hz}$, and $\omega_{\|}=2 \pi \times 40 \mathrm{~Hz}$, for the case of no DDI (black, dashed), $\theta=\pi / 2$ (blue, solid), and $\theta=0$ (red, dot-dashed). 


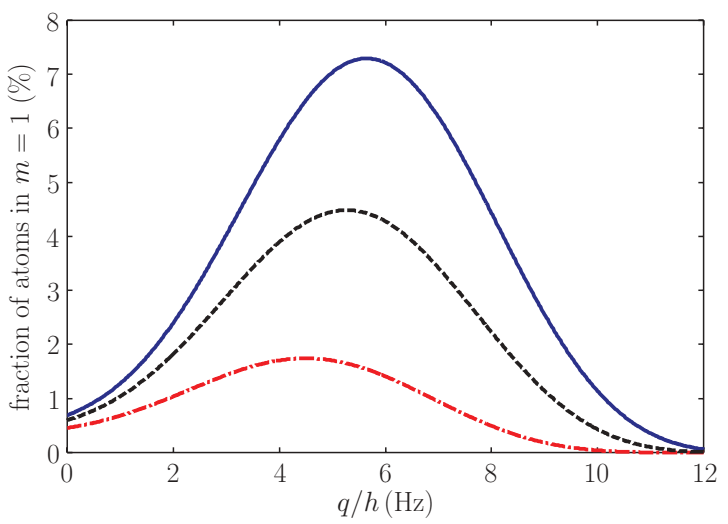

FIG. 2. (Color online) Fraction of atoms transferred into $| \pm 1\rangle$ after $115 \mathrm{~ms}$ as a function of $q$ for $N_{s}=2$ and the same parameters as Fig. 1, and for the case of no DDI (black, dashed), $\theta=\pi / 2$ (blue, solid), and $\theta=0$ (red, dot-dashed).

contrary to the homogeneous case. This maximum is induced by the inhomogeneous harmonic trapping and leads to marked resonances in the $q$ dependence of the amplification dynamics, as discussed in Ref. [21]. However, $\Lambda$ clearly depends on the trap orientation confirming indeed the intuitive qualitative picture discussed in Sec. IV. When trap axis and magnetic field are aligned $\Lambda$ decreases compared to the nondipolar case, whereas the opposite is true when the magnetic field is oriented perpendicular to the trap axis. Note as well that, as expected from the qualitative picture of Sec. IV, the instability region is shifted toward lower $q$ values in the parallel configuration, and toward larger $q$ values in the perpendicular one.

This modified instability rate translates into a significantly distorted pair-creation dynamics, due to the exponential nature of the parametric amplification. Figure 2 shows the transferred fraction $P_{ \pm 1}(t) / N$ after $t=115 \mathrm{~ms}$ as a function of $q$ for different values of $\theta$. As expected from the form of $\Lambda$ we observe the appearance of a maximum for all $\theta$, which is slightly shifted (by approximately $1 \mathrm{~Hz}$ ) toward lower $q$ when $\theta$ is shifted from $\pi / 2$ to 0 . However, this maximum is approximately four times as large for $\theta=\pi / 2$ than for $\theta=0$. The dependence of the amplification on $\theta$ is very clearly observable in the $\theta$ dependence of the maximum of $P_{ \pm 1}$ (again at $t=115 \mathrm{~ms}$ ) shown in Fig. 3. Note that the maximum grows monotonically from $\theta=0$ to $\theta=\pi / 2$.

\section{EFFECTS OF MAGNETIC-FIELD GRADIENTS ON THE AMPLIFICATION DYNAMICS}

As mentioned in Sec. II the homogeneous LZE plays typically no role in the spinor dynamics (only at very low magnetic fields $B<1 \mathrm{mG}$ the DDI could induce the equivalent of the Einstein-de Haas effect $[25,26]$, and in this case the residual LZE could play a role). However, magnetic-field gradients cannot be gauged out, and may play a relevant role in the spinor physics [27]. In this section, we analyze the effects that these gradients may have on the amplification dynamics. We shall show that even relatively weak gradients may have a significant effect on the amplification process.

Although magnetic-field gradients do not affect the GP equation for the $m=0 \mathrm{BEC}$, there is indeed a contribution

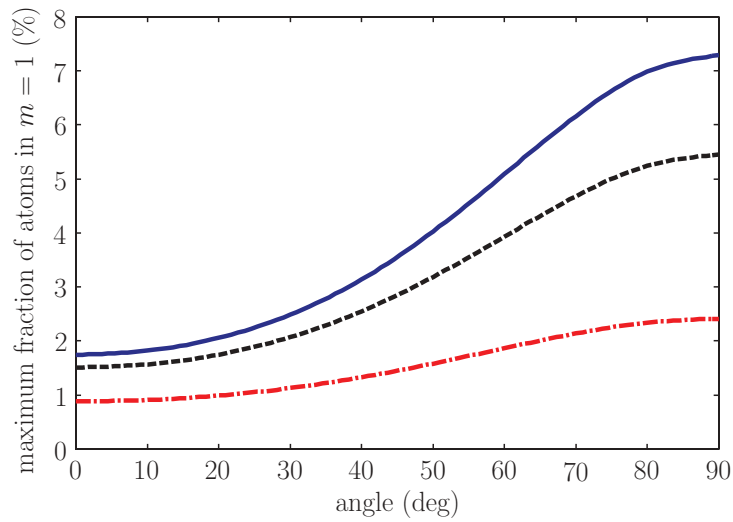

FIG. 3. (Color online) Maximal population transfer after $115 \mathrm{~ms}$ as a function of $\theta$ (same parameters as Fig. 2) for different magneticfield gradients $\nabla B=0$ (blue, solid), $5 \mathrm{mG} / \mathrm{cm}$ (black, dashed), and $10 \mathrm{mG} / \mathrm{cm}$ (red, dot-dashed).

to the effective Hamiltonian for $\delta \hat{\psi}_{ \pm 1}$ in the linear regime:

$$
\hat{H}_{1, \mathrm{gr}}=\vec{\nabla} p \cdot \int d^{3} r\left(\delta \hat{\psi}_{1}^{\dagger} \vec{r} \delta \hat{\psi}_{1}-\delta \hat{\psi}_{-1}^{\dagger} \vec{r} \delta \hat{\psi}_{-1}\right),
$$

which may be straightforwardly implemented into the matrix C of the eigenvalue equation (18),

$$
\mathbf{C}=\left[\begin{array}{cc}
\mathbf{E}+q \mathbf{1}+\mathbf{B}+\mathbf{D} & -\mathbf{A}+\mathbf{B} \\
\mathbf{A}+\mathbf{B} & -\mathbf{E}-q \mathbf{1}-\mathbf{B}+\mathbf{D}
\end{array}\right],
$$

with $D_{n n^{\prime}}=\vec{\nabla} p \cdot \int d^{3} r \phi_{n} \vec{r} \phi_{n^{\prime}}$.

The magnetic-field gradients have two main effects. On one side, they modify the effective potential $V_{\text {eff }}(\vec{r})$ in a different way for $m=1$ than for $m=-1$. This reduces the overlap of the $m= \pm 1$ atom clouds with the $m=0$ BEC and hence the scattering mediated transfer. On the other side, atoms placed at different locations experience different Larmor precession frequencies. Although this does not affect the local short-range interactions, it does modify the nonlocal DDI. For large-enough gradients this may lead to a time-averaged DDI [27]. For weak gradients, as those considered in the following, the explicit time dependence induced by the gradients must be considered.

Parametric amplification is handicapped by the presence of gradients as a result of these two combined effects. Figure 4 shows the combined effect of the DDI and the magnetic-field gradient along the weak trap axis for $\theta=\pi / 2$. As expected, we obtain a reduction of the transfer maximum with increasing gradient and a shift of its position to lower $q / h$ by approximately $1.5 \mathrm{~Hz}$ for a gradient of $10 \mathrm{mG} / \mathrm{cm}$. Hence the transfer maximum is shifted down and to lower $q$ with decreasing $\theta$ and increasing gradient. As shown in Fig. 3, in the presence of a magnetic-field gradient, the maximum of $P_{ \pm 1}(t)$ also shows a marked $\theta$ dependence.

Hence, even rather weak gradients $(<10 \mathrm{mG} / \mathrm{cm})$ may strongly modify the amplification dynamics, an effect which is enhanced by the presence of the DDI. Although as mentioned earlier, the $\theta$ dependence should also reveal in the presence of gradients the effects of the DDI, slight variations of the magnetic-field gradients (of the order of a few $\mathrm{mG} / \mathrm{cm}$ ) when changing the magnetic-field orientation with respect to the trap axis must be very carefully controlled. This is indeed 


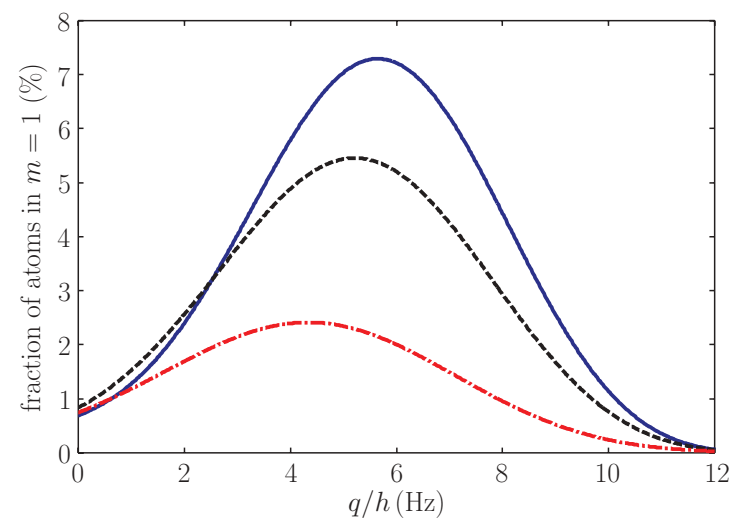

FIG. 4. (Color online) Fraction of atoms in \pm 1 after a time $115 \mathrm{~ms}$ (same parameters as Fig. 2) as a function of $q$ for $\theta=\pi / 2$, and for gradients $\vec{\nabla} B=\overrightarrow{0}$ (blue, solid), $5 \mathrm{mG} / \mathrm{cm}$ (black, dashed), and $10 \mathrm{mG} / \mathrm{cm}$ (red, dot-dashed).

a crucial point, since otherwise, accidental $\theta$ dependencies of the magnetic-field gradients may obscure the physical $\theta$ dependence characteristic of the DDI.

\section{DISCUSSION}

\section{A. Experimental requirements}

In the following we outline the general requirements to perform an experiment with $F=1{ }^{87} \mathrm{Rb}$ to probe the theoretical results discussed earlier. A $m=0$ BEC must be prepared in a crossed dipole trap following the same procedure detailed in Refs. [21,22]. The state preparation requires particular attention, since the remaining atoms in $m \neq 0$ may strongly alter the experimental result. Previous experiments have shown that the number of atoms in $m \neq 0$ states can be suppressed to $N_{s} \approx 2$ by briefly applying a strong magnetic-field gradient to purify the system [22]. Due to the nature of the QZE in $F=1{ }^{87} \mathrm{Rb}$, magnetic fields can be used to access positive values of $q$, as required previously.

As discussed in Sec. VII, the most significant requirement compared to previous experiments is related to the suppression of magnetic-field gradients which could obscure the dipolar effects. Appropriate experiments should be carefully designed to minimize all sources of field gradients from the vicinity of the atomic sample (e.g., a magnetic $\mu$-metal shielding could be placed around the sample). In state of the art precision measurements, field gradients are commonly suppressed below $1 \mathrm{mG} / \mathrm{cm}$ [29], which is sufficient to realize the mandatory experimental conditions (see Sec. VII).

Figure 2 shows that the resonant spin transfer to the $m= \pm 1$ states depends strongly on the relative orientation of the weak trap axis and the external magnetic field. Since it is difficult to change the orientation of a dipole trap while maintaining its trapping potential, experiments must be designed to vary the orientation of the external magnetic field. In this sense, two sets of Helmholtz coils are necessary to provide a homogeneous external magnetic field. One of them should be placed along the weak axis of the trapping potential to realize the $\theta=0$ configuration and another one along one of the strong axes to realize the $\theta=\pi / 2$ case. Both magnetic fields have to be calibrated, preferentially using precision microwave spectroscopy between the ground-state hyperfine manifolds of ${ }^{87} \mathrm{Rb}$. Such an experimental apparatus would also allow for a rotation of the field, since the currents in the two Helmholtz coils could be adjusted to obtain a relative angle $\theta$. In this way, it should be possible to perform a measurement analogous to that discussed in Fig. 3. Finally, additional magnetic-field gradients can be applied along both magnetic-field directions to observe the suppression shown in Fig. 4.

\section{B. Summary}

We have shown that, in spite of the very small magnetic moment, the magnetic DDI may lead to a strong modification of the amplification dynamics in $F=1{ }^{87} \mathrm{Rb}$ due to the low-energy scale of the spin-changing collisions. These effects must be carefully considered in all experiments dealing with parametric amplification in $F=1{ }^{87} \mathrm{Rb}$ (e.g., future experiments on number squeezing). In particular, we have shown that the DDI induce a very marked dependence of the amplification gain with respect to the relative orientation between magneticfield direction and trap axis. If both directions are perpendicular to each other the amplification dynamics is much faster than for the parallel configuration. Remarkably, the number of transferred atoms into $m= \pm 1$ may increase for $F=1{ }^{87} \mathrm{Rb}$ for a fixed holding time of around $100 \mathrm{~ms}$ by a factor over $400 \%$ when turning from a parallel to a perpendicular configuration. We have shown as well that magnetic-field gradients may also significantly modify the amplification dynamics, both due to their effects on the trapping and on the DDI. As a result, in order to cleanly reveal DDI effects in the parametric amplification in spinor condensates, magnetic-field gradients must be carefully controlled, since uncontrolled changes in the gradient when turning the magnetic-field orientation may obscure the orientation dependence of the DDI effects on the amplification. This demands specific requirements for future experiments in $F=1{ }^{87} \mathrm{Rb}$.

\section{ACKNOWLEDGMENTS}

We acknowledge support from the Centre for Quantum Engineering and Space-Time Research QUEST, from the Deutsche Forschungsgemeinschaft (SFB 407), and the European Science Foundation (EuroQUASAR).
[1] T.-L. Ho, Phys. Rev. Lett. 81, 742 (1998).

[2] T. Ohmi and K. Machida, J. Phys. Soc. Jpn. 67, 1822 (1998).

[3] M. Koashi and M. Ueda, Phys. Rev. Lett. 84, 1066 (2000).

[4] C. V. Ciobanu, S.-K. Yip, and T.-L. Ho, Phys. Rev. A 61, 033607 (2000).
[5] M.-S. Chang, C. D. Hamley, M. D. Barrett, J. A. Sauer, K. M. Fortier, W. Zhang, L. You, and M. S. Chapman, Phys. Rev. Lett. 92, 140403 (2004).

[6] H. Schmaljohann, M. Erhard, J. Kronjager, M. Kottke, S. vanStaa, L. Cacciapuoti, J. J. Arlt, K. Bongs, and K. Sengstock, Phys. Rev. Lett. 92, 040402 (2004). 
[7] L. E. Sadler et al., Nature (London) 443, 312 (2006).

[8] M. A. Baranov, Phys. Rep. 464, 71 (2008).

[9] T. Lahaye et al., Rep. Prog. Phys. 72, 126401 (2009).

[10] T. Lahaye et al., Nature (London) 448, 672 (2007).

[11] T. Koch et al., Nature Phys. 4, 218 (2008).

[12] T. Lahaye, J. Metz, B. Frohlich, T. Koch, M. Meister, A. Griesmaier, T. Pfau, H. Saito, Y. Kawaguchi, and M. Ueda, Phys. Rev. Lett. 101, 080401 (2008).

[13] J. Metz et al., New J. Phys. 11, 055032 (2009).

[14] M. Fattori, G. Roati, B. Deissler, C. DErrico, M. Zaccanti, M. Jona-Lasinio, L. Santos, M. Inguscio, and G. Modugno, Phys. Rev. Lett. 101, 190405 (2008).

[15] S. E. Pollack, D. Dries, M. Junker, Y. P. Chen, T. A. Corcovilos, and R. G. Hulet, Phys. Rev. Lett. 102, 090402 (2009).

[16] M. Vengalattore, S. R. Leslie, J. Guzman, and D. M. StamperKurn, Phys. Rev. Lett. 100, 170403 (2008).

[17] L.-M. Duan, A. Sorensen, J. I. Cirac, and P. Zoller, Phys. Rev. Lett. 85, 3991 (2000).

[18] H. Pu and P. Meystre, Phys. Rev. Lett. 85, 3987 (2000).

[19] D. F. Walls and G. Milburn, Quantum Optics (Springer, Heidelberg, 1994).
[20] S. R. Leslie, J. Guzman, M. Vengalattore, J. D. Sau, M. L. Cohen, and D. M. Stamper-Kurn, Phys. Rev. A 79, 043631 (2009).

[21] C. Klempt, O. Topic, G. Gebreyesus, M. Scherer, T. Henninger, P. Hyllus, W. Ertmer, L. Santos, and J. J. Arlt, Phys. Rev. Lett. 103, 195302 (2009).

[22] C. Klempt, O. Topic, G. Gebreyesus, M. Scherer, T. Henninger, P. Hyllus, W. Ertmer, L. Santos, and J. J. Arlt, Phys. Rev. Lett. 104, 195303 (2010).

[23] F. Gerbier, A. Widera, S. Folling, O. Mandel, and I. Bloch, Phys. Rev. A 73, 041602(R) (2006).

[24] L. Santos, M. Fattori, J. Stuhler, and T. Pfau, Phys. Rev. A 75, 053606 (2007).

[25] L. Santos and T. Pfau, Phys. Rev. Lett. 96, 190404 (2006).

[26] Y. Kawaguchi, H. Saito, and M. Ueda, Phys. Rev. Lett. 96, 080405 (2006).

[27] R. W. Cherng and E. Demler, Phys. Rev. Lett. 103, 185301 (2009).

[28] A. Lamacraft, Phys. Rev. Lett. 98, 160404 (2007).

[29] A. Gauguet, B. Canuel, T. Leveque, W. Chaibi, and A. Landragin, Phys. Rev. A 80, 063604 (2009). 